DESIGN CONSIDERATIONS FOR PERSONNEL ACCESS PENETRATIONS FOR THE SUPERCONDUCTING SUPER COLLIDER

J. D. Cossairt

April 1985 
1. Introduction

The detailed design of the tunnel for the SSC will, hopefully, soon begin. In this note are documented some considerations concerning the design of the radiological shielding for personnel access labyrinths. The methods are standard ones used at Fermilab, most recently described in detail (along with experimental results) in a paper by Cossairt, et.al. ${ }^{I}$ In the present paper a conceptual design is presented which will provide the required amount of protection for use of the SSC both as a pure collider and as an accelerator used for a fixed-target experimental program. A recipe will be developed which will allow designers to adjust these calculations for small deviations from this conceptual design.

2. Brief Summary of Computational Methods

It is customary in labyrinth calculations to calculate the dose or dose equivalent rate at the mouth of the labyrinth (the entrance to the tunnel fram the labyrinth) by either "rule of thumb" methods or more sophisticated techniques such as Monte-Carlo calculations using, for example, CASIM ${ }^{2}$. The attenuation of this dose or dose equivalent rate is then calculated for a given labyrinth relative to this "dose at the mouth". This has the advantage of separating the factor due to the source from the attenuation factor due to the various legs of the penetration. At the time of the design of the Fermilab labyrinths, a set of rather extensive calculations of neutron attenuation by various types of labyrinths were reported by Gollon and Awschalan using Monte-Carlo techniques ${ }^{3,4}$. The results indicated that it is possible to scale these calculations to labyrinths of different dimensions. In practice this is done for rectilinear labyrinth legs intersecting perpendicularly by choosing the linear coordinates to be in units of $\sqrt{A}$, where $A$ is the cross-sectional area of the enclosure. 
Recently, Tesch has found that rather simple formulae describe the attenuation characteristics of "people-sized" labyrinths ${ }^{5}$. In Ref. 1, results scaled from the Monte-Carlo calculations of Gollon and Awschalom and those obtained using a slightly modified version of Tesch's formulae are compared with absorbed dose rate attenuation data obtained at a proton energy of $400 \mathrm{GeV}$. Both methods of calculation agreed to within a factor of three with the data over a total attenuation corresponding to $10^{-5}$, measured for a four-legged labyrinth.

\section{Application to the SSC}

Figure 1 shows a possible design of such a penetration to the SSC tunnel. The radius of the tunnel cross section was chosen to be $1.5 \mathrm{~m}$ and the magnets were considered to be centered therein, since exact details are unknown at the present time. The earth overburden was chosen to be the minimum seven meters determined by bulk shielding requirements ${ }^{6}$. This is the situation which places the most severe constraints on the labyrinth design, since more deeply buried tunnels automatically guarantee longer vertical shafts. Experience with labyrinths at Fermilab and elsewhere has taught designers that segments which do not directly view the beam elements are much more effective neutron attenuators than so-called "first legs". Thus it is most efficient to make the first leg a horizontal segment and then use a vertical shaft to reach grade level, since this second leg is already constrained to be at least six meters long due to hadron shielding considerations. It is clear that the first leg should not be chosen to be excessively long, since the attenuation of it is approximately inverse square, i.e. goes as $r_{1}{ }^{2} / a^{2}$. Thus it is a less effective attenuator than are legs 2 and 3. which attenuate roughly as the sum of two exponentials. In fact, one of the results of Ref. $I$ is that the neutrons in leg $I$ have a higher average quality factor (QF) in leg I, (approx. 5.5) than in succeeding legs (approx. 3.4). The neutron spectrum in such a leg appears to have a rather energetic ("hard") 
component while that in successive sections is daminated by themal neutrons.

It is now necessary to calculate the dose equivalent at the "mouth", the entrance to the first leg. In 1983, Van Ginneken calculated the dose equivalent per proton lost at various locations in the soil shield surrounding a tunnel of one meter radius ${ }^{7}$. The largest dose equivalent calculated at the inner wall was approximately $2 \times 10^{-6} \mathrm{mrem} /$ proton lost. This peak value was obtained about $20 \mathrm{~m}$ downstream of the beam loss, assumed to be at a point in this case. Because of the longitudinal extent of the hadronic cascade, one should consider this to be a "line source". When scaling from a tunnel 1.0 meter in radius to one 1.5 meter in radius, the dose equivalent at the inner wall of the tunnel becomes $1.3 \times 10^{-6}$ mrem/proton. This calculation was done for $20 \mathrm{TeV}$ protons incident upon magnets reasonable in dimensions for the SSC.

Now one must define how much attenuation is needed. Since the SSC is so large, it is prudent to require the dose equivalent rate at the outside entrance to the labyrinth to be quite small, compatible with occupancy by members of the general public. For this reason, an annual dose equivalent limit of 10 mrem, either fram randam losses or fram infrequent (approx. once per year) large scale losses of beam was chosen to be the maximum allowable at the outside door. This is consistent with the criteria used in Ref. 6.

The most stringent case arises if the SSC is used to produce secondary beams for a fixed target experimental program, as has been discussed for other radiological problems in Ref. 6. In that paper it is clear that a reasonable loss pattern for randam beam scraping (18) might be of the order of $10^{12} \mathrm{~m}^{-1} \mathrm{yr}^{-1}$ while the "once per year" large scale accident might be 5 times higher. The large scale accident for the SSC used purely as a collider would also likely be of order $10^{12} \mathrm{~m}^{-1}$. The calculation reported in Ref. 7 shows that, at the wall, 
a $30 \mathrm{~m}$ length of tunnel is exposed to a dose greater than half of the peak value. Thus, it is prudent to take as a source factor the losses over a comparable length of tunnel so that the large scale accident for the SSC used to produce beans for a fixed target program would produce a dose of:

$$
\left[1.3 \times 10^{-6} \text { mrem } / \text { proton }\right] \times\left[5 \times 10^{12} \text { proton } / \mathrm{m}\right] \times 30 \mathrm{~m}=1.95 \times 10^{8} \text { mrem }
$$

The labyrinth thus needs to produce an attenuation in the dose equivalent of a factor of $5.13 \times 10^{-8}$.

Figures 2 and 3 give attenuation curves calculated for several plausible segments of labyrinths using the results of Ref. 2 and the adapted method of Tesch reported in Ref. 1. The longitudinal coordinates are defined as shown in Fig. 1. Figure 2 gives those calculated for the rather ineffective first leg for two different tunnel sizes, as indicated. It is clear that the method of Gollon and Awschalan is the more conservative for the initial segment while the method of Tesch is more conservative for succeeding legs. The latter gives attenuations only dependent upon the cross-sectional area of the laybrinth. Here are displayed results for two segments with circular cross sections defined by radius $R \quad(R=0.5 \mathrm{~m}$ and $R=0.7 \mathrm{~m})$ and two with rectangular cross sections. Once beyond the first leg, these results, as far as is known experimentally, could be applied to any number of succeeding legs. The designer of the labyrinth can then take these curves and calculate the lengths of segments necessary to achieve the desired attenuation. Prudence would suggest that taking the average of the two methods would be a reasonable procedure for obtaining meaningful estimates. The difference between this average and the calculations can be called the uncertainty. In the example shown here, the choice of the first leg to have a Im $x 2.1 \mathrm{~m}$ cross section and to be $4 \mathrm{~m}$ long $\left(r_{I}=5.5 \mathrm{~m}\right)$ will result in an attenuation of $0.11 \pm 0.03$. This leaves an attenuation factor of 


$$
(4.7 \pm 1.3) \times 10^{-7}
$$

to be achieved by the remaining segments. If the next leg is taken to be $a$ vertical cylinder with $R=0.7 \mathrm{~m}$ extending upward to a value of $r_{2}=6 \mathrm{~m}$, an attenuation of

$$
(2.0 \pm 1.4) \times 10^{-3}
$$

is achieved. This leaves an attenuation factor of

$$
(2.4 \pm 2.0) \times 10^{-4}
$$

to be achieved by the third leg. If it is assumed that the horizontal leg is a Im $\times 2.1 \mathrm{~m}$ horizontal segment and taking the average of the two calculations, it is found that the above value is reached at a value of $r_{3}=12 \mathrm{~m}$, with an uncertainty of $\pm 2 m$ based upon the differences between the two calculations. For conservatism, the choice of the longer final leg would be advisable, given the overall factor of three uncertainty in these methods. Of course, the third leg could be shortened by the addition of a fourth segment. Another aspect of the problem is the thickness of the earth overburden which must exist above the end of the vertical second segment. This overburden must provide a neutron attenuation equivalent to that of the thira leg. A result of the measurements of Ref. I was that the neutron spectrum in the second leg of such a penetration is soft, being daminated by thermal neutrons. Figure F. 6 of a report by the National Council on Radiation Protection and Measurements gives attenuation of dose equivalent by concrete for neutrons having energies between thermal and 100 $\mathrm{MeV}^{8}$. This reference indicates this attenuation to be slowly varying with energy from $10 \mathrm{MeV}$ down to themal energies. The required value of $2.4 \times 10^{-4}$ is provided by about $250 \mathrm{~g} / \mathrm{cm}^{2}$ of concrete, equivalent to $120 \mathrm{~cm}$ of soil having a density of $2.1 \mathrm{~g} / \mathrm{cm}$. Thus the earth overburden over the end of the second leg is rather modest, and is as shown in Fig. 1. 
4. Conclusion

Using existing methods, it will be possible to design effective personnel access penetrations for the SSC. As the design develops in detail, these procedures can be applied to its specific requirements.

The author would like to thank S. Baker for his helpful reading of this report. 
REFERENCES

1. J. D. Cossairt, J. G. Couch, A. J. Elwyn, and W. S. Freeman, "Radiation Measurements in a Labyrinth Penetration at a High Energy Proton Accelerator", Fermilab FN-4ø6, July, 1984 (to be published in Health Physics).

2. A. Van Ginneken and M. Awschalom, High Energy particle Interactions in Large Targets, Vol. 1 (Fermilab, Batavia, Illinois, 1975).

3. P. J. Gollon and M. Awschalom, "Design of Penetrations in Hadron Shields", IEEE Trans. Nucl. Sci. NS-18 (1971),

4. P. J. Gollon and M. Awschalom, "Design of Penetrations in Hadron Shields", CERN Report 71-16, Vol. 2, p. 697 (1971).

5. K. Tesch, "The Attenuation of the Neutron Dose Equivlent in a Labyrinth Through an Accelerator Shield", Particle Accelerators 12, 169 (1982).

6. J. D. Cossairt and A. J. Elwyn, "Shielding Considerations for Fixed Target Usage of the SSC", Proceedings of the SSC Fixed Target Workshop, p. 19. (The Woodlands, Texas, 1984).

7. A. Van Ginneken, memo to J. Bjorken and R. Huson, March 21, 1983, Also published in the Report of the $20 \mathrm{TeV}$ Hadron Collider Technical Workshop (Cornell University, March 28-April 2, 1983). 
8. "Radiation Protection Guidelines for 0.1-100 MeV Particle Accelerator Facilities", National Council on Radiation Protection and Measurements (NCRP Report No. 51, March 1, 1977). 
LIST OF EIGURE CAPTIONS

1. Plan and elevation views of a possible personnel access penetration for the SSC.

2. Attenuation curves for two examples of "first" legs plotted as a function of coordinate $r_{1}$ for calculations scaling from the results of Gollon and Awschalom (G-A) and by the equations in FN-406. For comparison, an inverse square law dependence is also shown.

3. Attenuation curves for legs succeeding the first plotted as a function of the coordinate $r_{I}(i>1)$. The notation is similar to that of Fig. 2 . 

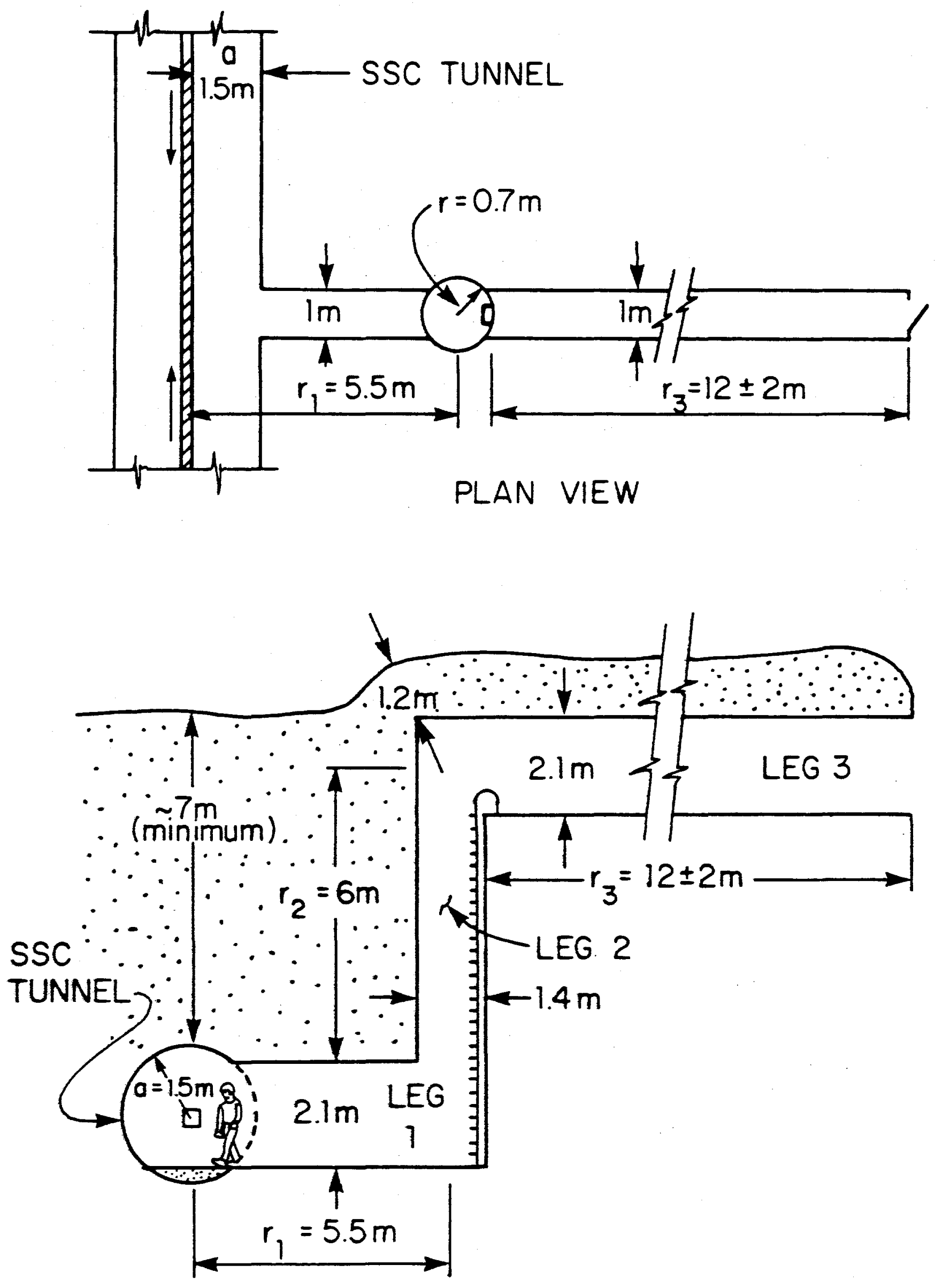

ELEVATION VIEW

Fig. 1 
FIRST LEG LABYRINTH ATTENUATION

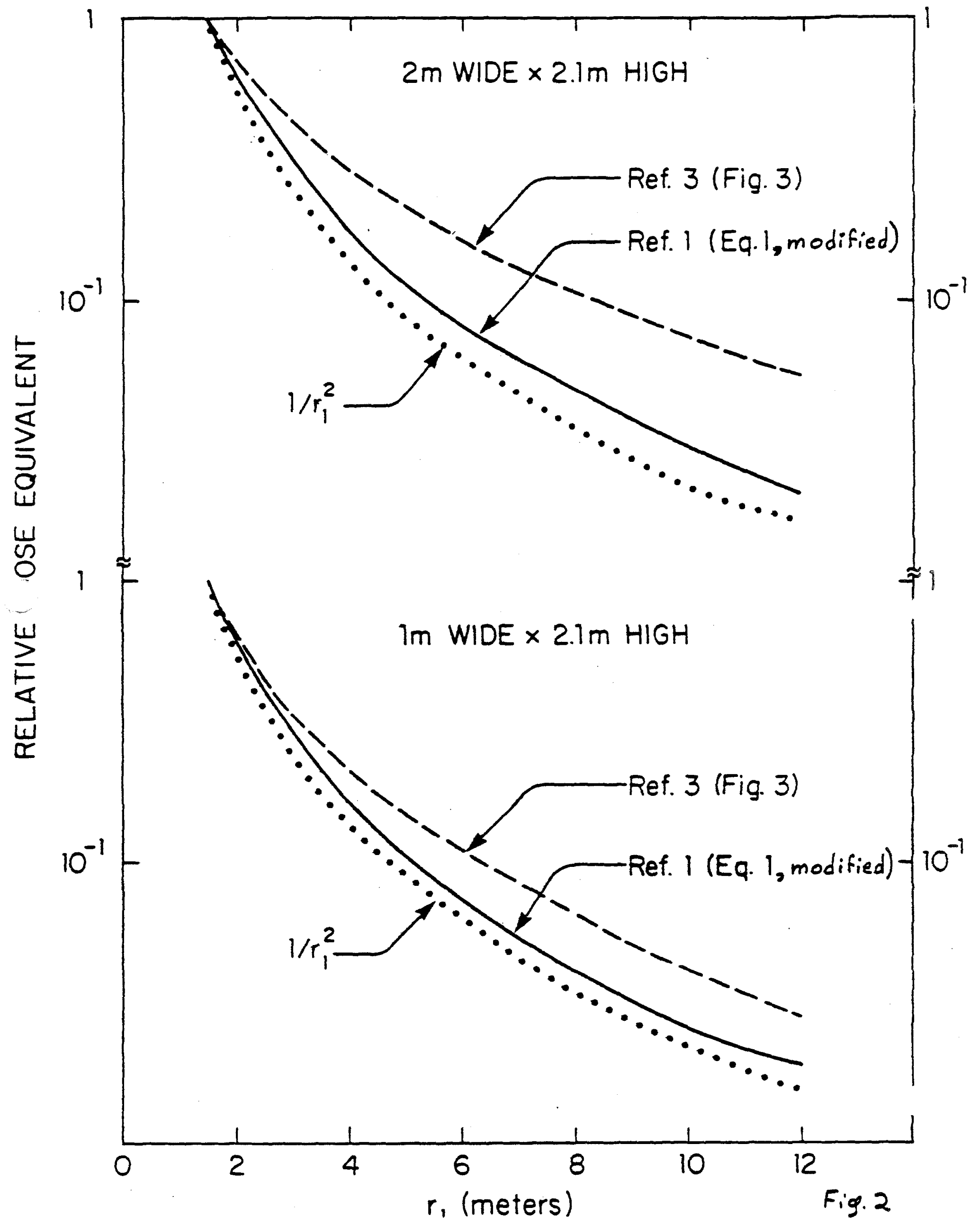




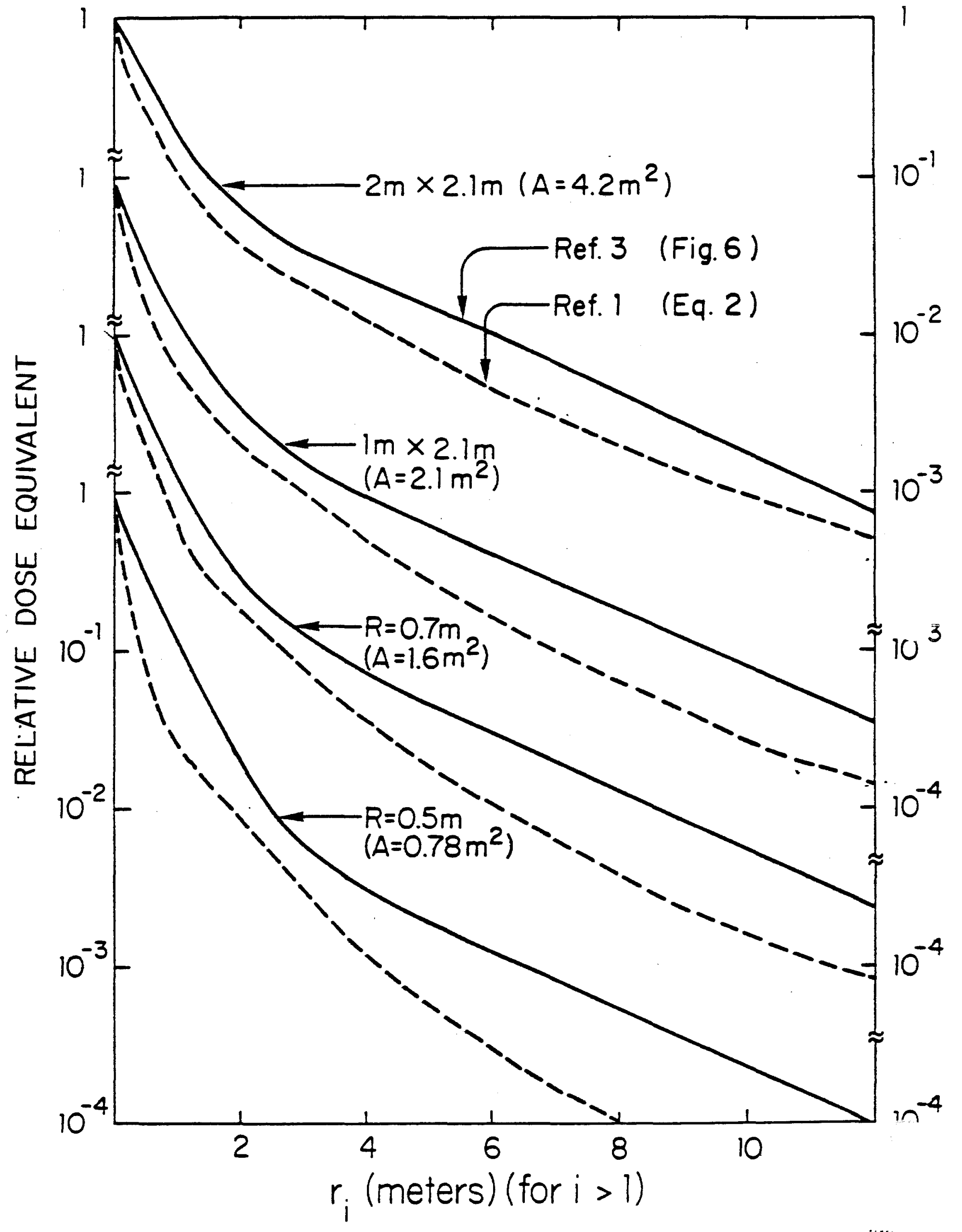

Fig. 3 